\title{
MODERATE DEVIATIONS FOR STABLE RANDOM WALKS IN RANDOM SCENERY
}

\author{
YUQIANG LI,* East China Normal University
}

\begin{abstract}
In this paper, a moderate deviation theorem for one-dimensional stable random walks in random scenery is proved. The proof relies on the analysis of maximum local times of stable random walks, and the comparison of moments between random walks in random scenery and self-intersection local times of the underlying random walks.
\end{abstract}

Keywords: Random walk in random scenery; stable random walk; moderate deviation

2010 Mathematics Subject Classification: Primary 60K37

Secondary 60F10

\section{Introduction}

Let $\xi_{1}, \xi_{2}, \ldots$ be a sequence of independent, identically distributed (i.i.d.) $\mathbb{Z}^{d}$-valued random vectors. A random walk $\left\{S_{n}, n \geq 0\right\}$ in $\mathbb{Z}^{d}$ with $S_{0}=0$ is defined as $S_{n}=\sum_{k=1}^{n} \xi_{k}$ for each $n \geq 1$. Let $\left\{\zeta_{i}, i \in \mathbb{Z}\right\}$ be a sequence of i.i.d. nondegenerate random variables taking values in $\mathbb{R}$. We refer to $\left\{\zeta_{i}, i \geq 0\right\}$ as the random scenery. Then the process $\left\{X_{n}, n \geq 1\right\}$ defined by

$$
X_{n}=\sum_{k=0}^{n-1} \zeta_{S_{k}}, \quad n \geq 1,
$$

is called a random walk in random scenery (with underlying random walk $\left\{S_{n}\right\}$ ). The random walk in random scenery is often rewritten as

$$
X_{n}=\sum_{x \in \mathbb{Z}^{d}} \zeta_{x} L_{n}(x), \quad n \geq 1,
$$

where $L_{n}(x)=\sum_{j=0}^{n-1} \mathbf{1}_{\{x\}}\left(S_{j}\right)$ is the local time of $\left\{S_{n}\right\}$ at $x$ before time $n$. Random walks in random scenery have a heuristic interpretation. If a random walker has to pay $Y_{z}$ units every time he/she visits the site $z$, then $X_{n}$ is the total amount he/she pays before time $n$.

A random walk in random scenery in the $d=1$ case was formally introduced and analyzed by Kesten and Spitzer [13]. They proved that if $\left\{\xi_{k}, k \geq 0\right\}$ and $\left\{\zeta_{x}, x \in \mathbb{Z}\right\}$ belong to domains of attraction of different stable laws with indices $1<\alpha \leq 2$ and $0<\beta \leq 2$, respectively, then $n^{-\delta} X_{n}$ converges in distribution as $n \rightarrow \infty$ to a nondegenerate variable, where $\delta=1-1 / \alpha+1 / \alpha \beta$. Since then there has been much work on random walks in scenery. For example, when $S_{n}$ is a simple random walk in $\mathbb{Z}^{d}$, Csáki et al. [8] studied the strong invariance principle for $X_{n}$ in the $d=2$ case, Asselah and Castell [2] estimated the probability that $X_{n}$ is large in the $d \geq 5$ case, and Asselah [1] investigated the moderate deviation for $X_{n}$ in the $d=3$ case. More generally, if the underlying random walks have finite variance, Gantert

Received 27 May 2010; revision received 9 October 2011.

* Postal address: School of Finance and Statistics, East China Normal University, Shanghai 200241, P. R. China.

Email address: yqli@stat.ecnu.edu.cn 
et al. [11] analyzed the deviations $\mathrm{P}\left(X_{n} / n>b_{n}\right)$ for various choices of sequences $\left\{b_{n}\right\}_{n \in \mathbb{N}}$ in $[1, \infty)$ with $b_{n} \rightarrow \infty$ as $n \rightarrow \infty$ in the case of arbitrary sceneries unbounded to $+\infty$ and Fleischmann et al. [10] proved moderate deviation principles for $X_{n}$ in the $d \geq 2$ case with the random sceneries satisfying Cramér's condition. In addition, when the underlying random walk has infinite variance, Lewis showed in [15] that a law of the iterated logarithm for $X_{n}$ can be obtained provided random normalizers are employed, and provided in [16] some sufficient conditions to obtain the deterministic normalizers.

We note that Khoshnevian and Lewis [14] proved a large deviation theorem for stable processes in Brownian scenery which, in essence, is the limit processes of random walks in random scenery under certain conditions. Their results (see [14, Theorems 1.1 and 5.1]) read as follows.

Theorem 1.1. ([14].) Suppose that $X=\left\{X_{t}: t \geq 0\right\}$ is a strictly stable Lévy processes with index $\alpha \in(1,2]$ and $Y=\{Y(t), t \in \mathbb{R}\}$ is a two-sided Brownian motion. Let $L=\left\{L_{t}^{x}: t \geq 0\right.$, $x \in \mathbb{R}\}$ denote the processes of local times of $X$. Define $G(t):=\int_{\mathbb{R}} L_{t}^{x} \mathrm{~d} Y(x)$. Then there exist a positive real number $\gamma_{1}=\gamma(\alpha)$ and some constant $\gamma_{2}>0$ such that

$$
\lim _{\lambda \rightarrow \infty} \lambda^{-2 \alpha /(1+\alpha)} \ln \mathrm{P}\left(G_{1} \geq \lambda\right)=-\gamma_{1}
$$

and

$$
\limsup _{t \rightarrow \infty}\left(\frac{\ln \ln t}{t}\right)^{1-1 / 2 \alpha} \frac{G(t)}{(\ln \ln t)^{3 / 2}}=\gamma_{2} \quad \text { almost surely. }
$$

Although the constants $\gamma_{1}$ and $\gamma_{2}$ are not specific, these results are still very interesting. It is natural to ask whether similar results hold for the random walks in random scenery when $\xi$. belongs to the domain of attraction of stable distributions and $\zeta$., as in, e.g. [8] and [11], is generally non-Gaussian. This question does not seem to have been addressed directly by Khoshnevian and Lewis' approach, since their proofs are heavily dependent on the properties of Gaussian processes and/or Brownian motions.

The purpose of this short paper is to partly solve the problem for a one-dimensional random walk in random scenery. We note that Chen [4], by means of the large deviation results of intersection local times of random walks in [6], investigated limit laws for the energy of a charged polymer and got interesting moderate deviation principles in different dimensional cases. Moreover, Chen and Khoshnevisan [5], under the assumption that the underlying random walk has finite second moments, pointed out that the model of polymers is close to random walks in random scenery. This result motivates us to study the problem by modifying the methods used in Chen [4], if we have large deviation principals for the self-intersection local times of the corresponding stable random walks.

The rest of this paper is organized as follows. In Section 2 we specify the assumptions and introduce the main result of this paper, and then prove a comparison lemma on moments between the random walk in random scenery and the self-intersection local times of underlying random walks. In Section 3 we prove in detail a limit theorem for the logarithmic moment generating function of the maximum local times of stable random walks. The proof of the main result is given in Section 4.

Throughout this paper, we use $\mathrm{E}^{\omega}$ to denote the expectation with respect to the scenery variables only, and $\mathrm{E}$ and $\mathrm{P}$ to respectively denote the expectation and probability with respect to both the random walk and scenery.

We use the notation $C$ and $C_{k}, k \geq 1$, to denote positive, finite constants, whose values can change at every occurrence, and which never depend on random quantities. 


\section{Main result}

Let $\xi_{1}, \xi_{2}, \ldots$ be nondegenerate, symmetric i.i.d. random variables taking values in $\mathbb{Z}$. Let $S_{0}=0$ and $S_{n}=\sum_{k=1}^{n} \xi_{k}$ for each $n \geq 1$. In the remainder of this paper, we suppose that $\left\{S_{n}\right\}$ is strongly aperiodic with support $\mathbb{Z}$ and assume that there exists $g(x)$, a function of regular variation with index $1 / \alpha$, such that $S_{n} / g(n) \rightarrow X$ in law as $n \rightarrow \infty$, where $\mathrm{E}\left(\mathrm{e}^{\mathrm{i} \lambda X}\right)=\mathrm{e}^{-|\lambda|^{\alpha}}$ and $\alpha \in(1,2]$. Then, the $\xi_{k}, k \geq 1$, belong to the domain of attraction of the symmetric $\alpha$-stable distribution, and, hence, in this paper we call $S=\left\{S_{n}\right\}$ the symmetric stable random walk (with $\alpha$ ) for convenience.

Let $L_{n}(x)$ be the local time of $\left\{S_{n}\right\}$ at $x$ before time $n$, i.e. $L_{n}(x)=\sum_{j=0}^{n-1} \mathbf{1}_{\{x\}}\left(S_{j}\right)$. From Theorem 4 of [7] we know that, for any positive sequence $\left\{b_{n}, n \geq 0\right\}$ with $b_{n} \rightarrow \infty$ and $b_{n} / n \rightarrow 0$,

$$
\lim _{n \rightarrow \infty} \frac{1}{b_{n}} \ln \mathrm{P}\left(\sum_{x \in \mathbb{Z}} L_{n}^{2}(x) \geq \lambda \frac{n^{2}}{g\left(n / b_{n}\right)}\right)=-\lambda^{\alpha} \frac{1}{2 \alpha}\left(\frac{2 \alpha-1}{2 \alpha M_{\alpha, 2}}\right)^{2 \alpha-1},
$$

where

$$
M_{\alpha, 2}=\sup _{f \in \mathcal{F}_{\alpha}}\left\{\|f\|_{4}^{2}-\int_{-\infty}^{\infty}|\lambda|^{\alpha}|\hat{f}(\lambda)|^{2} \mathrm{~d} \lambda\right\}<\infty
$$

$\hat{f}$ is the Fourier transform of $f$, and

$$
\mathcal{F}_{\alpha}=\left\{f \in L^{2}(\mathbb{R}):\|f\|_{2}=1 \text { and } \int_{-\infty}^{\infty}|\lambda|^{\alpha}|\hat{f}(\lambda)|^{2} \mathrm{~d} \lambda<\infty\right\} .
$$

For simplicity, we always assume in the sequel that

$$
\sigma:=\lim _{x \rightarrow \infty} x^{-1 / \alpha} g(x)>0 .
$$

Therefore, from (2.1), we obtain

$$
\lim _{n \rightarrow \infty} \frac{1}{b_{n}} \ln \mathrm{P}\left(\sum_{x \in \mathbb{Z}} L_{n}^{2}(x) \geq \lambda \frac{n^{2}}{\left(n / b_{n}\right)^{1 / \alpha}}\right)=-\lambda^{\alpha} \frac{\sigma^{\alpha}}{2 \alpha}\left(\frac{2 \alpha-1}{2 \alpha M_{\alpha, 2}}\right)^{2 \alpha-1} .
$$

For convenience, in the rest of this paper, let

$$
C_{\alpha}:=\sigma^{\alpha}\left(\frac{2 \alpha-1}{2 \alpha M_{\alpha, 2}}\right)^{2 \alpha-1} .
$$

Consider the stable random walk in random scenery

$$
X_{n}=\sum_{k=0}^{n-1} \zeta_{S_{k}}=\sum_{x \in \mathbb{Z}} \zeta_{x} L_{n}(x)
$$

where the random scenery $\zeta=\left\{\zeta_{x}, x \in \mathbb{Z}\right\}$, independent of $\left\{S_{n}, n \geq 0\right\}$, is a family of nondegenerate, symmetric, i.i.d. real-valued random variables satisfying Equation (1.2) of [4], i.e.

$$
\mathrm{E}\left(\zeta_{1}^{2}\right)=1 \quad \text { and } \quad \mathrm{E}\left(\mathrm{e}^{\lambda_{0} \zeta_{1}^{2}}\right)<\infty \quad \text { for some } \lambda_{0}>0
$$

Our main result is a moderate deviation theorem for $X_{n}$. 
Theorem 2.1. Under the above assumptions,

$$
\lim _{n \rightarrow \infty} \frac{1}{b_{n}} \ln \mathrm{P}\left( \pm X_{n} \geq \lambda n^{1-1 / 2 \alpha} b_{n}^{1 / 2+1 / 2 \alpha}\right)=-\frac{\alpha+1}{\alpha} \lambda^{2 \alpha /(\alpha+1)}\left(\frac{C_{\alpha}}{2 \sqrt{8^{\alpha}}}\right)^{1 /(\alpha+1)}
$$

for any positive sequence $\left\{b_{n}\right\}$ satisfying

$$
b_{n} \rightarrow \infty \text { and } b_{n}=o\left(n^{1 /(2 \alpha+1)}\right) .
$$

Remark 2.1. Theorem 2.1 generalizes the corresponding result in [14] (see (1.1)) to random walks in general random sceneries. Furthermore, we specify that

$$
\gamma_{1}=\frac{\alpha+1}{\alpha}\left(\frac{C_{\alpha}}{2 \sqrt{8^{\alpha}}}\right)^{1 /(\alpha+1)} .
$$

The proof of Theorem 2.1 is similar to that of Theorem 1.2 of [4] with some necessary modifications to handle the technical complexities caused by the stable random walks. The basic idea is to compare the moments between the random walks in random scenery and the self-intersection local times of underlying random walks by using localization. See [4] for more details and [6] for some related tricks.

In the rest of this section we develop an analogue of Proposition 2.1 of [4] to compare the moments of the localized random walks in random scenery with those of the corresponding self-intersection local times. Recall that the self-intersection local times of $S=\left\{S_{n}\right\}$ are

$$
H_{n}=\sum_{x \in \mathbb{Z}} L_{n}^{2}(x)
$$

For positive constants $K_{n}=M_{n} n^{1-1 / \alpha} b_{n}^{1 / \alpha}>0$, where

$$
M_{n} \rightarrow \infty \text { and } \frac{M_{n}^{3 \alpha} b_{n}^{2 \alpha+1}}{n} \rightarrow 0
$$

as $n \rightarrow \infty$, define

$$
\begin{aligned}
& \tilde{X}_{n}=X_{n} \mathbf{1}_{\left\{\sup _{x \in \mathbb{Z}} L_{n}(x) \leq K_{n}\right\}}, \\
& \tilde{H}_{n}=H_{n} \mathbf{1}_{\left\{\sup _{x \in \mathbb{Z}} L_{n}(x) \leq K_{n}\right\}},
\end{aligned}
$$

and

$$
A_{m}(n)=\sum_{\left(y_{1}, y_{2}, \ldots, y_{m}\right) \in B_{m}} \mathrm{E}\left(\mathbf{1}_{\left\{\sup _{x \in \mathbb{Z}} L_{n}(x) \leq K_{n}\right\}} \prod_{k=1}^{m} L_{n}^{2}\left(y_{k}\right)\right),
$$

where $m, n=1,2, \ldots$ and $B_{m}=\left\{\left(y_{1}, \ldots, y_{m}\right) \in \mathbb{Z}^{m} ; y_{1}, \ldots, y_{m}\right.$ are distinct $\}$. By applying similar arguments used in the proof of Proposition 2.1 of [4] we obtain the following result.

Lemma 2.1. There exists a constant $C$ independent of $n, m$, and the choice of $K_{n}$ such that

$$
\mathrm{E}\left(\tilde{X}_{n}^{m}\right) \leq m ! \sum_{l=1}^{[m / 2]} \frac{1}{l !} 2^{-l / 2} K_{n}^{m-2 l} C^{(m-2 l) / 2}\left(\begin{array}{c}
m-l-1 \\
m-2 l
\end{array}\right) \mathrm{E}\left(\tilde{H}_{n}^{l}\right) .
$$

On the other hand, for any integers $m, n \geq 1$,

$$
\mathrm{E}\left(\tilde{X}_{n}^{2 m}\right) \geq \frac{(2 m !)}{2^{m} m !} A_{m}(n)
$$


and

$$
\mathrm{E}\left(\tilde{H}_{n}^{m}\right) \leq \sum_{l=1}^{m}\left(\begin{array}{c}
m \\
l
\end{array}\right) l^{m-l} K_{n}^{2(m-l)} A_{l}(n)
$$

Proof. As the proof follows the same lines as that of Proposition 2.1 of [4] with some modifications, to save space, we only show the modification necessary for the proof of (2.5).

By replacing $\Lambda_{n}(x)$ in Equation (2.10) of [4, p. 644] with $2 \zeta_{x} L_{n}(x)$ and applying the same arguments that lead to Equation (2.14) of [4, p. 646], we obtain

$$
\begin{aligned}
\mathrm{E}\left(\tilde{X}_{n}^{m}\right)= & \sum_{l=1}^{\left[2^{-1} m\right]} \frac{1}{l !} \sum_{\substack{i_{1}+\cdots+i_{l}=m \\
i_{1}, \ldots, i_{l} \geq 2}} \frac{m !}{\left(i_{1}\right) ! \cdots\left(i_{l}\right) !} \\
& \times \sum_{\left(y_{1}, \ldots, y_{m}\right) \in B_{l}} \mathrm{E}\left(\mathbf{1}_{\left\{\sup _{x \in \mathbb{Z}} L_{n}(x) \leq K_{n}\right\}} \prod_{k=1}^{l} L_{n}\left(y_{k}\right)^{i_{k}} \mathrm{E}^{\omega}\left(\zeta_{y_{k}}^{i_{k}}\right)\right) .
\end{aligned}
$$

Condition (2.3) implies that there exists a constant $C_{1}>1$ such that

$$
\mathrm{E}\left(\zeta_{x}^{i}\right) \leq\left(\mathrm{E}\left(\zeta_{x}^{2 i}\right)\right)^{1 / 2} \leq\left(i ! C_{1}^{i}\right)^{1 / 2}
$$

for all $i \geq 3$. Therefore, from (2.8) we obtain

$$
\begin{aligned}
\mathrm{E}\left(\tilde{X}_{n}^{m}\right) \leq & m ! \sum_{l=1}^{\left[2^{-1} m\right]} \frac{1}{l !} \sum_{\substack{i_{1}+\cdots+i_{l}=m \\
i_{1}, \ldots, i_{l} \geq 2}} \frac{C_{i_{1}}^{i_{1} / 2} \cdots C_{i_{l}}^{i_{l} / 2}}{\sqrt{\left(i_{1}\right) ! \cdots\left(i_{l}\right) !}} \\
& \times \sum_{\left(y_{1}, \ldots, y_{m}\right) \in B_{l}} \mathrm{E}\left(\mathbf{1}_{\left\{\sup _{x \in \mathbb{Z}} L_{n}(x) \leq K_{n}\right\}} \prod_{k=1}^{l} L_{n}\left(y_{k}\right)^{i_{k}}\right),
\end{aligned}
$$

where $C_{2}=1$ and $C_{k}=C_{1}$ for $k \geq 3$. Since

$$
\mathbf{1}_{\left\{\sup _{x \in \mathbb{Z}} L_{n}(x) \leq K_{n}\right\}} \prod_{k=1}^{l} L_{n}\left(y_{k}\right)^{i_{k}} \leq K_{n}^{m-2 l} \mathbf{1}_{\left\{\sup _{x \in \mathbb{Z}} L_{n}(x) \leq K_{n}\right\}} \prod_{k=1}^{l} L_{n}\left(y_{k}\right)^{2},
$$

when $i_{1}+\cdots+i_{l}=m$ and $i_{1}, \ldots, i_{l} \geq 2$, we further have

$$
\begin{aligned}
\mathrm{E}\left(\tilde{X}_{n}^{m}\right) & \leq m ! \sum_{l=1}^{\left[2^{-1} m\right]} \frac{1}{l !} \sum_{\substack{i_{1}+\cdots+i_{l}=m \\
i_{1}, \ldots, i_{l} \geq 2}} \frac{C_{i_{1}}^{i_{1} / 2} \cdots C_{i_{l}}^{i_{l} / 2}}{\sqrt{\left(i_{1}\right) ! \cdots\left(i_{l}\right) !}} K_{n}^{m-2 l} \mathrm{E}\left(\tilde{H}^{l}\right) \\
& \leq m ! \sum_{l=1}^{\left[2^{-1} m\right]} \frac{2^{-l / 2}}{l !} K_{n}^{m-2 l} \mathrm{E}\left(\tilde{H}^{l}\right) \sum_{\substack{i_{1}+\cdots+i_{l}=m \\
i_{1}, \ldots, i_{l} \geq 2}} C_{i_{1}}^{i_{1} / 2} \cdots C_{i_{l}}^{i_{l} / 2} .
\end{aligned}
$$

Let $k$ be the number of elements in the set $\left\{0 \leq j \leq l, i_{j}=2\right\}$. Then $m \geq 2 k+3(l-k)$, 
which leads to $l-k \leq m-2 l$. Therefore,

$$
\begin{aligned}
\sum_{\substack{i_{1}+\cdots+i_{l}=m \\
i_{1}, \ldots, i_{l} \geq 2}} C_{i_{1}}^{i_{1} / 2} \cdots C_{i_{l}}^{i_{l} / 2} & \leq \sum_{\substack{i_{1}+\cdots+i_{l}=m \\
i_{1}, \ldots, i_{l} \geq 2}} C_{1}^{(m-2 k) / 2} \\
& =\sum_{\substack{i_{1}+\cdots+i_{l}=m \\
i_{1}, \ldots, i_{l} \geq 2}} C_{1}^{(m-2 l+2 l-2 k) / 2} \\
& \leq \sum_{\substack{i_{1}+\cdots+i_{l}=m \\
i_{1}, \ldots, i_{l} \geq 2}} C_{1}^{3(m-2 l) / 2} \\
& =C^{(m-2 l) / 2}\left(\begin{array}{c}
m-l-1 \\
m-2 l
\end{array}\right)
\end{aligned}
$$

where $C=C_{1}^{3}$. Substituting (2.10) into (2.9) yields (2.5).

Remark 2.2. It is readily seen that $\tilde{X}_{n}$ is symmetric, and, hence, by $(2.5), \mathrm{E}\left(\tilde{X}_{n}^{2 m+1}\right)=0$ for each integer $m \geq 0$.

\section{Deviations of the maximum local time of stable random walks}

In this section we prove a limit theorem for the logarithmic moment generating function of the maximum local times of the symmetric stable random walk $S_{n}$. This is not only an important step in the proof of the main result, but is also of independent interest. The main result of this section is as follows.

Theorem 3.1. There exists a constant $C>0$ such that, for any $\lambda>0$ and positive sequence $\left\{b_{n}\right\}$ with $b_{n} \rightarrow \infty$ and $b_{n}=o(n)$ as $n \rightarrow \infty$,

$$
\limsup _{n \rightarrow \infty} \frac{1}{b_{n}} \ln \mathrm{E}\left(\exp \left\{\lambda\left(\frac{b_{n}}{n}\right)^{1-1 / \alpha} \sup _{x} L_{n}(x)\right\}\right)<\frac{1}{2}(2 C \lambda \vee 1)^{\alpha /(\alpha-1)}<+\infty .
$$

In the case where the random walks have finite second moment, the finiteness of the lim sup in (3.1) seems to be well known; it was used by Chen [4] without specific proof. Our result deals with the case of stable random walks and provides an explicit constant as the upper bound. The proof of the theorem is based on the refinements of some results in [12] and Chen's ideas on local times (see [3]). To avoid unnecessary repetition with the material included in [12], identical parts are omitted.

For convenience, let $c_{n}:=\left(n / b_{n}\right)^{1 / \alpha}$ in this section.

The following lemma generalizes Lemma 9 of [12].

Lemma 3.1. There is a constant $C>0$ such that if $|x-y| \leq \eta c_{n}$ then

$$
\mathrm{P}\left(\frac{c_{n}}{n}\left|L_{n}(x)-L_{n}(y)\right| \geq C \eta^{(\alpha-1) / 4}\right) \leq \eta^{b_{n}(\alpha-1) / 2}
$$

for all $n, 0<\eta<1$.

Proof. By the same arguments as those used in the proof of the first part of Lemma 9 of [12] we obtain, for some constant $C>0$,

$$
\mathrm{E}\left[\left(L_{n}(x)-L_{n}(y)\right)^{2 r}\right] \leq(2 r) ! C^{r}\left(m a_{m}^{-1}\right)^{r}(|x-y| Q(|x-y|))^{-r},
$$


where $m=[n / r]+1, Q(x)=\mathrm{E}\left[\left(x^{-1}\left|\xi_{1}\right| \wedge 1\right)^{2}\right]$ for $x \geq 0$, and $a_{x}$ satisfies $Q\left(a_{x}\right)=1 / x$ for $x>1 / Q(1)$ and $a_{x}=1$ for $x \in[0,1 / Q(1)]$. Note that $\xi_{1}$ is in the domain of attraction of an $\alpha$-stable law with $\alpha \in(1,2]$. There exists a constant $C_{1}>0$ such that $\lim _{x \rightarrow \infty} x^{\alpha} Q(x)=C_{1}$. Therefore, for any $1 \leq x \leq y, x^{\alpha} Q(x) \geq C_{2} y^{\alpha} Q(y)$ for some constant $C_{2}$ and $a_{n}=O\left(n^{1 / \alpha}\right)$. Now, since $|x-y| \leq \eta c_{n}$, we obtain

$$
|x-y| Q(|x-y|) \geq C_{2} \eta^{-(\alpha-1)} c_{n} Q\left(c_{n}\right),
$$

and, hence,

$$
\begin{aligned}
\mathrm{E}\left[\left(\frac{c_{n}}{n}\left(L_{n}(x)-L_{n}(y)\right)\right)^{2 r}\right] & \leq\left(\frac{C}{C_{2}}\right)^{r}(2 r)^{2 r}\left(\frac{c_{n}}{n}\right)^{2 r}\left(\frac{m}{a_{m}}\right)^{r}\left(\frac{1}{c_{n} Q\left(c_{n}\right)}\right)^{r} \eta^{r(\alpha-1)} \\
& \leq C_{3}^{r} r^{2 r}\left(\frac{c_{n}}{n}\right)^{2 r}\left(\frac{n}{r}\right)^{(1-1 / \alpha) r} c_{n}^{(\alpha-1) r} \eta^{r(\alpha-1)} \\
& =C_{3}^{r} r^{r+r / \alpha} c_{n}^{r+\alpha r} \frac{1}{n^{r+r / \alpha}} \eta^{r(\alpha-1)} \\
& =C_{3}^{r}\left(\frac{r}{b_{n}}\right)^{r+r / \alpha} \eta^{r(\alpha-1)} .
\end{aligned}
$$

Let $r=b_{n}$. Then

$$
\mathrm{E}\left[\left(\frac{c_{n}}{n}\left(L_{n}(x)-L_{n}(y)\right)\right)^{2 b_{n}}\right] \leq C_{3}^{b_{n}} \eta^{b_{n}(\alpha-1)} .
$$

Using Markov's inequality, we obtain

$$
\begin{aligned}
\mathrm{P}\left(\frac{c_{n}}{n}\left|L_{n}(x)-L_{n}(y)\right| \geq \sqrt{C_{3}} \eta^{(\alpha-1) / 4}\right) & \leq \frac{1}{C_{3}^{b_{n}} \eta^{b_{n}(\alpha-1) / 2}} \mathrm{E}\left[\left(\frac{c_{n}}{n}\left(L_{n}(x)-L_{n}(y)\right)\right)^{2 r}\right] \\
& \leq \eta^{b_{n}(\alpha-1) / 2},
\end{aligned}
$$

which is the desired conclusion if we take $C=\sqrt{C_{3}}$.

Lemma 3.2. For any $\varepsilon>0$, there exist a constant $0<\delta<1$ independent of $\left\{c_{n}\right\}$ and a constant $n_{0}>0$ such that

$$
\mathrm{P}\left(\sup _{|x-y| \leq \delta c_{n}} \frac{c_{n}}{n}\left|L_{n}(x)-L_{n}(y)\right| \geq \varepsilon\right) \leq \mathrm{e}^{-b_{n}} \quad \text { for all } n>n_{0} .
$$

Proof. Since the random walk is $\alpha$-stable with $\alpha \in(1,2]$, by Lemma 3 of [12], there exist a constant $C>0$ and $\lambda>1$ such that $\mathrm{P}\left(\max _{1 \leq k \leq n}\left|S_{k}\right| \geq M a_{n}\right) \leq C M^{-\lambda}$ for all $n$ and $M>1$. Therefore,

$$
\mathrm{P}\left(L_{n}(x) \neq 0 \text { for some }|x| \geq \mathrm{e}^{2 b_{n}} a_{n}\right) \leq C \mathrm{e}^{-2 \lambda b_{n}}<C \mathrm{e}^{-2 b_{n}} .
$$

Let $\gamma=2^{-(\alpha-1) / 4}$. Choose $\delta<\mathrm{e}^{-16 /(\alpha-1)}$ such that $C \delta^{(\alpha-1) / 4} \leq \varepsilon(1-\gamma)$, where $C$ is the constant in Lemma 3.1, and let $j=\min \left\{k, \delta c_{n} \leq 2^{k}\right\}$. For $0<x<2^{j} \leq 2 \delta c_{n}<c_{n}$, we may write $x=\sum_{i=0}^{j} \chi_{i} 2^{i}$, where each $\chi_{i}=0$ or 1 . Let $x_{m}=\sum_{i=m}^{j} \chi_{i} 2^{i}$. By the same arguments used in the proof of Lemma 11 of [12] (see the last three lines on page 79), from Lemma 3.1 
we obtain

$$
\begin{aligned}
\mathrm{P}\left(\max _{0<x \leq \delta c_{n}} \frac{c_{n}}{n}\left|L_{n}(0)-L_{n}(x)\right| \geq \varepsilon\right) \\
\quad \leq \mathrm{P}\left(\max _{0<x \leq 2^{j}} \frac{c_{n}}{n}\left|L_{n}(0)-L_{n}(x)\right| \geq \varepsilon\right) \\
\quad \leq \sum_{m=0}^{j} 2^{j-m} \mathrm{P}\left(\frac{c_{n}}{n}\left|L_{k}\left(x_{m+1}\right)-L_{k}\left(x_{m}\right)\right| \geq \varepsilon \gamma^{j-m}(1-\gamma)\right) \\
\quad \leq \sum_{m=0}^{j} 2^{j-m} \mathrm{P}\left(\frac{c_{n}}{n}\left|L_{k}\left(x_{m+1}\right)-L_{k}\left(x_{m}\right)\right| \geq C\left(2^{m-j} \delta\right)^{(\alpha-1) / 4}\right) \\
\quad \leq \sum_{m=0}^{j} 2^{j-m}\left(2^{m-j} \delta\right)^{b_{n}(\alpha-1) / 2} .
\end{aligned}
$$

Note that $b_{n} \rightarrow \infty$ implies that

$$
\sum_{m=0}^{j} 2^{(m-j)\left(b_{n}(\alpha-1) / 2-1\right)} \leq 2
$$

for sufficiently large $n$. Therefore,

$$
\mathrm{P}\left(\max _{0<x \leq \delta c_{n}} \frac{c_{n}}{n}\left|L_{k}(0)-L_{k}(x)\right| \geq \varepsilon\right) \leq 2 \delta^{b_{n}(\alpha-1) / 2}
$$

for sufficiently large $n$. Since there are at most $2\left(\mathrm{e}^{2 b_{n}} a_{n}+c_{n}\right) / \delta c_{n}+2$ disjoint short intervals of length $\delta c_{n}$ in $\left[-\left(\mathrm{e}^{2 b_{n}} a_{n}+c_{n}\right), \mathrm{e}^{2 b_{n}} a_{n}+c_{n}\right]$, by the same arguments used in the proof of Lemma 11 of [12] (see pages 79 and 80), there exist constants $C_{1}, C_{2}>0$ which are independent of $c_{n}$ and $\delta$ such that

$$
\begin{aligned}
\mathrm{P}\left(\sup _{|x-y| \leq \delta c_{n}} \frac{c_{n}}{n}\left|L_{n}(x)-L_{n}(y)\right| \geq \varepsilon\right) \\
\quad \leq C \mathrm{e}^{-2 b_{n}}+C_{1} \delta^{b_{n}(\alpha-1) / 2} \frac{\mathrm{e}^{2 b_{n}} a_{n}+c_{n}}{\delta c_{n}} \\
\quad \leq C_{2}\left(\mathrm{e}^{-2 b_{n}}+\delta^{b_{n}(\alpha-1) / 2-1} \mathrm{e}^{2 b_{n}} b_{n}^{1 / \alpha}\right) \\
\quad=C_{2}\left(\mathrm{e}^{-2 b_{n}}+\exp \left\{b_{n}\left(\frac{\ln b_{n}}{\alpha b_{n}}+\left(\frac{\alpha-1}{2}-\frac{1}{b_{n}}\right) \ln \delta+2\right)\right\}\right)
\end{aligned}
$$

Since $b_{n} \rightarrow \infty$, we can choose suitable $n_{0}$ such that, for $n>n_{0}, 2 C_{2} \mathrm{e}^{-b_{n}}<1$ and

$$
\frac{\ln b_{n}}{\alpha b_{n}}+\left(\frac{\alpha-1}{2}-\frac{1}{b_{n}}\right) \ln \delta+2 \leq \frac{\ln b_{n}}{\alpha b_{n}}+\left(\frac{\alpha-1}{2}-\frac{1}{b_{n}}\right) \frac{-16}{\alpha-1}+2 \leq-2 .
$$

Consequently, for all $n \geq n_{0}$,

$$
\mathrm{P}\left(\sup _{|x-y| \leq \delta c_{n}} \frac{c_{n}}{n}\left|L_{n}(x)-L_{n}(y)\right| \geq \varepsilon\right) \leq 2 C_{2} \mathrm{e}^{-2 b_{n}} \leq \mathrm{e}^{-b_{n}},
$$

which completes the proof. 
Lemma 3.3. For sufficiently large n,

$$
\mathrm{P}\left(\frac{c_{n}}{n} \sup _{x} L_{n}(x) \geq \frac{1}{\delta}+\varepsilon\right) \leq \mathrm{e}^{-b_{n}},
$$

where $\delta$ and $\varepsilon$ are the constants in Lemma 3.2.

Proof. Suppose that, for some $n>n_{0}$ with $c_{n}>2 / \delta$,

$$
\mathrm{P}\left(\frac{c_{n}}{n} \sup _{x} L_{n}(x) \geq \frac{1}{\delta}+\varepsilon\right)>\mathrm{e}^{-b_{n}} .
$$

Then Lemma 3.2 implies that

$$
\begin{aligned}
& \mathrm{P}\left(\frac{c_{n}}{n} \sup _{x} L_{n}(x) \geq \frac{1}{\delta}+\varepsilon, \sup _{|x-y| \leq \delta c_{n}} \frac{c_{n}}{n}\left|L_{n}(x)-L_{n}(y)\right|<\varepsilon\right) \\
& \quad \geq \mathrm{P}\left(\frac{c_{n}}{n} \sup _{x} L_{n}(x) \geq \frac{1}{\delta}+\varepsilon\right)-\mathrm{P}\left(\sup _{|x-y| \leq \delta c_{n}} \frac{c_{n}}{n}\left|L_{n}(x)-L_{n}(y)\right| \geq \varepsilon\right) \\
& \quad \geq \mathrm{P}\left(\frac{c_{n}}{n} \sup _{x} L_{n}(x) \geq \frac{1}{\delta}+\varepsilon\right)-\mathrm{e}^{-b_{n}} \\
& \quad>0 .
\end{aligned}
$$

Note that

$$
\begin{aligned}
\left\{\frac{c_{n}}{n} \sup _{x} L_{n}(x) \geq \frac{1}{\delta}+\varepsilon, \sup _{|x-y| \leq \delta c_{n}} \frac{c_{n}}{n}\left|L_{n}(x)-L_{n}(y)\right|<\varepsilon\right\} \\
\subset\left\{\sum_{x \in \mathbb{Z}} \frac{c_{n}}{n} L_{n}(x) \geq \frac{2}{\delta}\left(\delta c_{n}-1\right)\right\} \\
\subset\left\{\sum_{x \in \mathbb{Z}} \frac{c_{n}}{n} L_{n}(x)>c_{n}\right\} .
\end{aligned}
$$

It follows that

$$
\mathrm{P}\left(\sum_{x \in \mathbb{Z}} \frac{c_{n}}{n} L_{n}(x)>c_{n}\right)>0
$$

which contradicts the fact that

$$
\sum_{x \in \mathbb{Z}} \frac{c_{n}}{n} L_{n}(x)=c_{n}
$$

Therefore, for any $n>\max \left\{n_{0}, \min \left\{n: c_{n}>2 / \delta\right\}\right\}$, (3.2) holds.

Lemma 3.4. For any nonnegative integer $r$, there exists a positive constant $C$ independent of $\left\{c_{n}\right\}$ such that

$$
\mathrm{E}\left[\left(\frac{c_{n}}{n} \sup _{x} L_{n}(x)\right)^{r}\right] \leq C^{r}\left(1+\frac{r !}{b_{n}^{r}}\right) .
$$

Proof. By Lemma 1 of [3] we obtain, for any $a, b>0$,

$$
\mathrm{P}\left(\frac{c_{n}}{n} \sup _{x} L_{n}(x)>a+b\right) \leq \mathrm{P}\left(\frac{c_{n}}{n} \sup _{x} L_{n}(x)>a\right) \mathrm{P}\left(\frac{c_{n}}{n} \sup _{x} L_{n}(x)>b\right) .
$$


Therefore, we can infer from Lemma 3.3 that

$$
F(u):=\mathrm{P}\left(\frac{c_{n}}{n} \sup _{x} L_{n}(x)>u\right) \leq\left(\mathrm{e}^{-b_{n}}\right)^{\left[u / C_{1}\right]} \leq \mathrm{e}^{-b_{n}\left(u / C_{1}-1\right)}
$$

for all $u>C_{1}:=1 / \delta+\varepsilon$. Hence,

$$
\begin{aligned}
\mathrm{E}\left[\left(\frac{c_{n}}{n} \sup _{x} L_{n}(x)\right)^{r}\right] & =\int_{0}^{\infty} r x^{r-1} F(x) \mathrm{d} x \\
& \leq\left(2 C_{1}\right)^{r}+\int_{2 C_{1}}^{\infty} r x^{r-1} \mathrm{e}^{-b_{n}\left(x / C_{1}-1\right)} \mathrm{d} x \\
& \leq\left(2 C_{1}\right)^{r}+\int_{0}^{\infty} r x^{r-1} \mathrm{e}^{-b_{n} x / 2 C_{1}} \mathrm{~d} x \\
& =\left(2 C_{1}\right)^{r}+\frac{r !}{\left(b_{n} / 2 C_{1}\right)^{r}} \\
& \leq\left(2 C_{1}\right)^{r}\left(1+\frac{r !}{b_{n}^{r}}\right) .
\end{aligned}
$$

Letting $C=2 C_{1}$ completes the proof.

Proof of Theorem 3.1. If $\lambda \leq 1 / 2 C$, where $C$ is the constant in Lemma 3.4, then, by Lemma 3.4 and Taylor's expansion, for any $\left\{b_{n}\right\}$ satisfying the conditions in Theorem 3.1 and sufficiently large $n$, we have

$$
\begin{aligned}
\ln \mathrm{E}\left(\exp \left\{\lambda\left(\frac{b_{n}}{n}\right)^{1-1 / \alpha} \sup _{x} L_{n}(x)\right\}\right) & =\ln \mathrm{E}\left(\exp \left\{\lambda b_{n} \frac{c_{n}}{n} \sup _{x} L_{n}(x)\right\}\right) \\
& =\ln \sum_{k=0}^{\infty} \frac{\left(\lambda b_{n}\right)^{k}}{k !} \mathrm{E}\left[\left(\frac{c_{n}}{n} \sup _{x} L_{n}(x)\right)^{k}\right] \\
& \leq \ln \sum_{k=0}^{\infty} \frac{\left(\lambda b_{n}\right)^{k}}{k !}\left(C^{k}+C^{k} \frac{k !}{b_{n}^{k}}\right) \\
& =\ln \left(\mathrm{e}^{\lambda C b_{n}}+\frac{1}{1-\lambda C}\right) .
\end{aligned}
$$

If $\lambda>1 / 2 C$ then

$$
\begin{aligned}
& \ln \mathrm{E}\left(\exp \left\{\lambda\left(\frac{b_{n}}{n}\right)^{1-1 / \alpha} \sup _{x} L_{n}(x)\right\}\right) \\
& \quad=\ln \mathrm{E}\left(\exp \left\{\frac{1}{2 C}\left(\frac{b_{n}(2 C \lambda)^{\alpha /(\alpha-1)}}{n}\right)^{1-1 / \alpha} \sup _{x} L_{n}(x)\right\}\right) .
\end{aligned}
$$

Letting $b_{n}^{\prime}=b_{n}(2 C \lambda)^{\alpha /(\alpha-1)}$, from (3.4) we obtain

$$
\ln \mathrm{E}\left(\exp \left\{\lambda\left(\frac{b_{n}}{n}\right)^{1-1 / \alpha} \sup _{x} L_{n}(x)\right\}\right) \leq \ln \left(\mathrm{e}^{(2 C \lambda)^{\alpha /(\alpha-1)} b_{n} / 2}+2\right)
$$

for sufficiently large $n$. From (3.4) and (3.5), it is easy to obtain the desired conclusion. This completes the proof. 


\section{Proof of the main result}

In this section we complete the proof of Theorem 2.1. Although this part is in essence the same as the proof in [4, pp. 651-655], we make some modifications owing to the stable random walks and introduce some simplifications by using the symmetry of the sceneries.

Owing to the fact that $H_{n} \leq n \sup _{x \in \mathbb{Z}} L_{n}(x)$ for any $\lambda>0$,

$$
\mathrm{E}\left(\exp \left\{\lambda \frac{b_{n}^{1-1 / \alpha}}{n^{2-1 / \alpha}} H_{n}\right\}\right) \leq \mathrm{E}\left(\exp \left\{\lambda\left(\frac{b_{n}}{n}\right)^{1-1 / \alpha} \sup _{x \in \mathbb{Z}} L_{n}(x)\right\}\right),
$$

which, together with Theorem 3.1, implies that

$$
\lim _{n \rightarrow \infty} \frac{1}{b_{n}} \ln \mathrm{E}\left(\exp \left\{\lambda \frac{b_{n}^{1-1 / \alpha}}{n^{2-1 / \alpha}} H_{n}\right\}\right)<+\infty .
$$

Therefore, by Varadhan's integral lemma (see Theorem 4.3.1 of [9, p. 137]), it follows from (2.2) and (4.1) that

$$
\lim _{n \rightarrow \infty} \frac{1}{b_{n}} \ln \mathrm{E}\left(\exp \left\{\lambda \frac{b_{n}^{1-1 / \alpha}}{n^{2-1 / \alpha}} H_{n}\right\}\right)=\sup _{y>0}\left\{y \lambda-\frac{C_{\alpha}}{2 \alpha} y^{\alpha}\right\}=\frac{\alpha-1}{2 \alpha} C_{\alpha}\left(\frac{2 \lambda}{C_{\alpha}}\right)^{\alpha /(\alpha-1)} .
$$

The symmetry of $\tilde{X}_{n}$ yields

$$
\mathrm{E}\left(\exp \left\{-\theta \frac{b_{n}^{1 / 2-1 / 2 \alpha}}{n^{1-1 / 2 \alpha}} \tilde{X}_{n}\right\}\right)=\mathrm{E}\left(\exp \left\{\theta \frac{b_{n}^{1 / 2-1 / 2 \alpha}}{n^{1-1 / 2 \alpha}} \tilde{X}_{n}\right\}\right)
$$

and, by Taylor's expansion and (2.5),

$$
\begin{aligned}
& \mathrm{E}\left(\exp \left\{\theta \frac{b_{n}^{1 / 2-1 / 2 \alpha}}{n^{1-1 / 2 \alpha}} \tilde{X}_{n}\right\}\right) \\
& \quad=1+\sum_{k=2}^{\infty} \frac{\theta^{k}}{k !}\left(\frac{b_{n}^{1 / 2-1 / 2 \alpha}}{n^{1-1 / 2 \alpha}}\right)^{k} \mathrm{E}\left(\tilde{X}_{n}^{k}\right) \\
& \quad \leq 1+\sum_{k=2}^{\infty} \frac{\theta^{k}}{k !}\left(\frac{b_{n}^{1 / 2-1 / 2 \alpha}}{n^{1-1 / 2 \alpha}}\right)^{k} k ! \sum_{l=1}^{[k / 2]} \frac{1}{l !} 2^{-l / 2} K_{n}^{k-2 l} C^{(k-2 l) / 2}\left(\begin{array}{c}
k-l-1 \\
k-2 l
\end{array}\right) \mathrm{E}\left(\tilde{H}_{n}^{l}\right)
\end{aligned}
$$

Furthermore, for any $\theta>0$ and sufficiently large $n$,

$$
\begin{aligned}
& \mathrm{E}\left(\exp \left\{\theta \frac{b_{n}^{1 / 2-1 / 2 \alpha}}{n^{1-1 / 2 \alpha}} \tilde{X}_{n}\right\}\right) \\
& \leq 1+\sum_{l=1}^{\infty} \frac{\theta^{2 l}}{l !}\left(\frac{b_{n}^{1 / 2-1 / 2 \alpha}}{n^{1-1 / 2 \alpha}}\right)^{2 l} 2^{-l / 2} \mathrm{E}\left(\tilde{H}_{n}^{l}\right) \\
& \times \sum_{k=2 l}^{\infty} \theta^{k-2 l}\left(\frac{b_{n}^{1 / 2-1 / 2 \alpha}}{n^{1-1 / 2 \alpha}}\right)^{k-2 l} K_{n}^{k-2 l} C^{(k-2 l) / 2}\left(\begin{array}{c}
k-l-1 \\
k-2 l
\end{array}\right) \\
& =1+\sum_{l=1}^{\infty} \frac{\theta^{2 l}}{l !}\left(\frac{b_{n}^{1 / 2-1 / 2 \alpha}}{n^{1-1 / 2 \alpha}}\right)^{2 l} 2^{-l / 2}\left(1-\frac{\sqrt{C} \theta K_{n} b_{n}^{1 / 2-1 / 2 \alpha}}{n^{1-1 / 2 \alpha}}\right)^{-(l-1)} \mathrm{E}\left(\tilde{H}_{n}^{l}\right) \\
& \leq \mathrm{E}\left(\exp \left\{\frac{\theta^{2}}{\sqrt{2}} \frac{b_{n}^{1-1 / \alpha}}{n^{2-1 / \alpha}}\left(1-\frac{\sqrt{C} \theta K_{n} b_{n}^{1 / 2-1 / 2 \alpha}}{n^{1-1 / 2 \alpha}}\right)^{-1} \tilde{H}_{n}\right\}\right)
\end{aligned}
$$


where we have used the facts that

$$
(1-x)^{-(l-1)}=\sum_{k=0}^{\infty}\left(\begin{array}{c}
l-1+k \\
k
\end{array}\right) x^{k}
$$

for $|x|<1$, and, as $n \rightarrow \infty$,

$$
\frac{\sqrt{C} \theta K_{n} b_{n}^{1 / 2-1 / 2 \alpha}}{n^{1-1 / 2 \alpha}}=\frac{\sqrt{C} \theta M_{n} b_{n}^{1 / 2+1 / 2 \alpha}}{n^{1 / 2 \alpha}} \rightarrow 0
$$

by (2.4). Combining (4.4) with (4.3) and (4.2) yields

$$
\limsup _{n \rightarrow \infty} \frac{1}{b_{n}} \mathrm{E}\left(\exp \left\{ \pm \theta \frac{b_{n}^{1 / 2-1 / 2 \alpha}}{n^{1-1 / 2 \alpha}} \tilde{X}_{n}\right\}\right) \leq \frac{\alpha-1}{2 \alpha} C_{\alpha}\left(\frac{\sqrt{2} \theta^{2}}{C_{\alpha}}\right)^{\alpha /(\alpha-1)} .
$$

On the other hand, from Remark 2.2 and Taylor's expansion, it follows that

$$
\mathrm{E}\left(\exp \left\{ \pm \theta \frac{b_{n}^{1 / 2-1 / 2 \alpha}}{n^{1-1 / 2 \alpha}} \tilde{X}_{n}\right\}\right)=1+\sum_{k=1}^{\infty} \frac{\theta^{2 k}}{(2 k) !}\left(\frac{b_{n}^{1 / 2-1 / 2 \alpha}}{n^{1-1 / 2 \alpha}}\right)^{2 k} \mathrm{E}\left(\tilde{X}_{n}^{2 k}\right)
$$

By (2.6),

$$
\sum_{k=1}^{\infty} \frac{\theta^{2 k}}{(2 k) !}\left(\frac{b_{n}^{1 / 2-1 / 2 \alpha}}{n^{1-1 / 2 \alpha}}\right)^{2 k} \mathrm{E}\left(\tilde{X}_{n}^{2 k}\right) \geq \sum_{k=1}^{\infty} \frac{1}{k !} \frac{\theta^{2 k}}{2^{k}}\left(\frac{b_{n}^{1 / 2-1 / 2 \alpha}}{n^{1-1 / 2 \alpha}}\right)^{2 k} A_{k}(n) .
$$

Letting

$$
\bar{\theta}=\theta \exp \left\{-\theta^{2} \frac{K_{n}^{2} b_{n}^{1-1 / \alpha}}{2 n^{2-1 / \alpha}}\right\} \leq \theta
$$

by (2.7) we have

$$
\begin{aligned}
\sum_{k=1}^{\infty} \frac{\bar{\theta}^{2 k}}{2^{k} k !}\left(\frac{b_{n}^{1 / 2-1 / 2 \alpha}}{n^{1-1 / 2 \alpha}}\right)^{2 k} \mathrm{E}\left(\tilde{H}_{n}^{k}\right) \\
\quad \leq \sum_{k=1}^{\infty} \frac{\bar{\theta}^{2 k}}{2^{k} k !}\left(\frac{b_{n}^{1 / 2-1 / 2 \alpha}}{n^{1-1 / 2 \alpha}}\right)^{2 k} \sum_{l=1}^{k}\left(\begin{array}{l}
k \\
l
\end{array}\right) l^{k-l} K_{n}^{2(k-l)} A_{l}(n) \\
\quad=\sum_{l=1}^{\infty} \frac{\bar{\theta}^{2 l}}{2^{l} l !}\left(\frac{b_{n}^{1 / 2-1 / 2 \alpha}}{n^{1-1 / 2 \alpha}}\right)^{2 l} A_{l}(n) \sum_{k=l}^{\infty} \frac{l^{k-l}}{(k-l) !} K_{n}^{2(k-l)}\left(\frac{b_{n}^{1 / 2-1 / 2 \alpha}}{n^{1-1 / 2 \alpha}}\right)^{2(k-l)}\left(\frac{\bar{\theta}^{2}}{2}\right)^{k-l} \\
\quad=\sum_{l=1}^{\infty} \frac{\bar{\theta}^{2 l}}{2^{l} l !}\left(\frac{b_{n}^{1 / 2-1 / 2 \alpha}}{n^{1-1 / 2 \alpha}}\right)^{2 l} A_{l}(n) \exp \left\{\bar{\theta}^{2} \frac{l K_{n}^{2} b_{n}^{1-1 / \alpha}}{2 n^{2-1 / \alpha}}\right\} \\
\leq \sum_{l=1}^{\infty} \frac{1}{2^{l} l !}\left(\frac{b_{n}^{1 / 2-1 / 2 \alpha}}{n^{1-1 / 2 \alpha}}\right)^{2 l} A_{l}(n)\left(\bar{\theta}^{2} \exp \left\{\theta^{2} \frac{K_{n}^{2} b_{n}^{1-1 / \alpha}}{2 n^{2-1 / \alpha}}\right\}\right)^{l} .
\end{aligned}
$$

Therefore,

$$
\sum_{k=1}^{\infty} \frac{\bar{\theta}^{2 k}}{2^{k} k !}\left(\frac{b_{n}^{1 / 2-1 / 2 \alpha}}{n^{1-1 / 2 \alpha}}\right)^{2 k} \mathrm{E}\left(\tilde{H}_{n}^{k}\right) \leq \sum_{l=1}^{\infty} \frac{\theta^{2 l}}{2^{l} l !}\left(\frac{b_{n}^{1 / 2-1 / 2 \alpha}}{n^{1-1 / 2 \alpha}}\right)^{2 l} A_{l}(n),
$$


which, together with (4.7), yields

$$
\begin{aligned}
1+\sum_{k=1}^{\infty} \frac{\theta^{2 k}}{(2 k) !}\left(\frac{b_{n}^{1 / 2-1 / 2 \alpha}}{n^{1-1 / 2 \alpha}}\right)^{2 k} \mathrm{E}\left(\tilde{X}_{n}^{2 k}\right) & \geq 1+\sum_{k=1}^{\infty} \frac{\bar{\theta}^{2 k}}{2^{k} k !}\left(\frac{b_{n}^{1 / 2-1 / 2 \alpha}}{n^{1-1 / 2 \alpha}}\right)^{2 k} \mathrm{E}\left(\tilde{H}_{n}^{k}\right) \\
& =\mathrm{E}\left(\exp \left\{\frac{\bar{\theta}^{2}}{2} \frac{b_{n}^{1-1 / \alpha}}{n^{2-1 / \alpha}} \tilde{H}_{n}\right\}\right) .
\end{aligned}
$$

Note that $\tilde{H}_{n} \leq n K_{n}$ and that

$$
\frac{K_{n}^{3} b_{n}^{1-1 / \alpha}}{n^{2-1 / \alpha}} \frac{b_{n}^{1-1 / \alpha}}{n^{1-1 / \alpha}}=\frac{M_{n}^{3} b_{n}^{2+1 / \alpha}}{n^{1 / \alpha}} \rightarrow 0
$$

by (2.4). We have

$$
\begin{aligned}
\frac{\bar{\theta}^{2}}{2} \frac{b_{n}^{1-1 / \alpha}}{n^{2-1 / \alpha}} \tilde{H}_{n} & =\frac{\theta^{2}}{2} \frac{b_{n}^{1-1 / \alpha}}{n^{2-1 / \alpha}} \tilde{H}_{n}-\frac{\theta^{2}}{2}\left[1-\exp \left\{-\theta^{2} \frac{K_{n}^{2} b_{n}^{1-1 / \alpha}}{n^{2-1 / \alpha}}\right\}\right] \frac{b_{n}^{1-1 / \alpha}}{n^{2-1 / \alpha}} \tilde{H}_{n} \\
& \geq \frac{\theta^{2}}{2} \frac{b_{n}^{1-1 / \alpha}}{n^{2-1 / \alpha}} \tilde{H}_{n}-\frac{\theta^{4}}{2} \frac{K_{n}^{3} b_{n}^{1-1 / \alpha}}{n^{2-1 / \alpha}} \frac{b_{n}^{1-1 / \alpha}}{n^{1-1 / \alpha}} \\
& =\frac{\theta^{2}}{2} \frac{b_{n}^{1-1 / \alpha}}{n^{2-1 / \alpha}} \tilde{H}_{n}-o(1) .
\end{aligned}
$$

Combining (4.6) with (4.7)-(4.9), we further obtain

$$
\mathrm{E}\left(\exp \left\{ \pm \theta \frac{b_{n}^{1 / 2-1 / 2 \alpha}}{n^{1-1 / 2 \alpha}} \tilde{X}_{n}\right\}\right) \geq \mathrm{E}\left(\exp \left\{\frac{\theta^{2}}{2} \frac{b_{n}^{1-1 / \alpha}}{n^{2-1 / \alpha}} \tilde{H}_{n}\right\}\right)-o(1)
$$

Observe that

$$
\begin{aligned}
\mathrm{E}\left(\exp \left\{\frac{\theta^{2}}{2} \frac{b_{n}^{1-1 / \alpha}}{n^{2-1 / \alpha}} \tilde{H}_{n}\right\}\right) \\
\geq \mathrm{E}\left(\exp \left\{\frac{\theta^{2}}{2} \frac{b_{n}^{1-1 / \alpha}}{n^{2-1 / \alpha}} H_{n}\right\} \mathbf{1}_{\left.\left\{\sup _{x \in \mathbb{Z}} L_{n}(x) \leq K_{n}\right\}\right)}\right. \\
=\mathrm{E}\left(\exp \left\{\frac{\theta^{2}}{2} \frac{b_{n}^{1-1 / \alpha}}{n^{2-1 / \alpha}} H_{n}\right\}\right)-\mathrm{E}\left(\exp \left\{\frac{\theta^{2}}{2} \frac{b_{n}^{1-1 / \alpha}}{n^{2-1 / \alpha}} H_{n}\right\} \mathbf{1}_{\left\{\sup _{x \in \mathbb{Z}} L_{n}(x)>K_{n}\right\}}\right) .
\end{aligned}
$$

By the Cauchy-Schwarz inequality,

$$
\begin{aligned}
\frac{1}{b_{n}} \ln & \mathrm{E}\left(\exp \left\{\frac{\theta^{2}}{2} \frac{b_{n}^{1-1 / \alpha}}{n^{2-1 / \alpha}} H_{n}\right\} \mathbf{1}_{\left\{\sup _{x \in \mathbb{Z}} L_{n}(x)>K_{n}\right\}}\right) \\
\leq & \frac{1}{b_{n}} \ln \left[\mathrm{E}\left(\exp \left\{\theta^{2} \frac{b_{n}^{1-1 / \alpha}}{n^{2-1 / \alpha}} H_{n}\right\}\right)^{1 / 2} \mathrm{P}\left(\sup _{x \in \mathbb{Z}} L_{n}(x)>K_{n}\right)^{1 / 2}\right] \\
& =\frac{1}{2 b_{n}} \ln \mathrm{E}\left(\exp \left\{\theta^{2} \frac{b_{n}^{1-1 / \alpha}}{n^{2-1 / \alpha}} H_{n}\right\}\right)+\frac{1}{2 b_{n}} \ln \mathrm{P}\left(\sup _{x \in \mathbb{Z}} L_{n}(x)>K_{n}\right) .
\end{aligned}
$$

In addition, since $K_{n}=M_{n} n^{1-1 / \alpha} b_{n}^{1 / \alpha}$ and $M_{n} \rightarrow \infty$, by (3.3) we obtain

$$
\frac{1}{2 b_{n}} \ln \mathrm{P}\left(\sup _{x \in \mathbb{Z}} L_{n}(x)>K_{n}\right) \leq \frac{1}{2 b_{n}} \ln \mathrm{e}^{-\left[M_{n} / C_{1}\right] b_{n}} \rightarrow-\infty,
$$


which, together with (4.1) and (4.12), implies that

$$
\frac{1}{b_{n}} \ln \mathrm{E}\left(\exp \left\{\frac{\theta^{2}}{2} \frac{b_{n}^{1-1 / \alpha}}{n^{2-1 / \alpha}} H_{n}\right\} \mathbf{1}_{\left\{\sup _{x \in \mathbb{Z}} L_{n}(x)>K_{n}\right\}}\right) \rightarrow-\infty .
$$

Consequently, it follows from (4.11) and (4.14) that

$$
\begin{aligned}
\liminf _{n \rightarrow \infty} \frac{1}{b_{n}} \ln \mathrm{E}\left(\exp \left\{\frac{\theta^{2}}{2} \frac{b_{n}^{1-1 / \alpha}}{n^{2-1 / \alpha}} \tilde{H}_{n}\right\}\right) & \geq \liminf _{n \rightarrow \infty} \frac{1}{b_{n}} \ln \mathrm{E}\left(\exp \left\{\frac{\theta^{2}}{2} \frac{b_{n}^{1-1 / \alpha}}{n^{2-1 / \alpha}} H_{n}\right\}\right) \\
& =\frac{\alpha-1}{2 \alpha} C_{\alpha}\left(\frac{\sqrt{2} \theta^{2}}{C_{\alpha}}\right)^{\alpha /(\alpha-1)} .
\end{aligned}
$$

Combining (4.5), (4.10), and (4.15), we obtain

$$
\lim _{n \rightarrow \infty} \mathrm{E}\left(\exp \left\{ \pm \theta \frac{b_{n}^{1 / 2-1 / 2 \alpha}}{n^{1-1 / 2 \alpha}} \tilde{X}_{n}\right\}\right)=\frac{\alpha-1}{2 \alpha} C_{\alpha}\left(\frac{\sqrt{2} \theta^{2}}{C_{\alpha}}\right)^{\alpha /(\alpha-1)}
$$

According to the Gärtner-Ellis theorem (see [9, Theorem 2.3.6, p. 44]), (4.16) implies that $\tilde{X}_{n}$ satisfies the moderate deviation given in Theorem 2.1. By [9, Theorem 4.2.13, p. 130], the moderate deviation passes from $\tilde{X}_{n}$ to $X_{n}$ through the exponential equivalence given by

$$
\limsup _{n \rightarrow \infty} \frac{1}{b_{n}} \ln \mathrm{P}\left(\tilde{X}_{n} \neq X_{n}\right)=\lim _{n \rightarrow \infty} \frac{1}{b_{n}} \ln \mathrm{P}\left(\sup _{x \in \mathbb{Z}} L_{n}(x)>K_{n}\right)=-\infty,
$$

which follows from (4.13). The proof is complete.

\section{Acknowledgements}

The author is grateful to the anonymous referee for suggestions which have helped to significantly improve the paper. This research was supported by the National Natural Science Foundation of China (grant number 10901054).

\section{References}

[1] Asselah, A. (2008). Large deviations estimates for self-intersection local times for simple random walk in $\mathbb{Z}^{3}$. Prob. Theory Relat. Fields 141, 19-45.

[2] Asselah, A. and Castell, F. (2007). Random walk in random scenery and self-intersection local times in dimensions $d \geq 5$. Prob. Theory Relat. Fields 138, 1-32.

[3] Chen, X. (2000). On the law of the iterated logarithm for local times of recurrent random walks. In High Dimensional Probability (Seattle, WA, 1999; Progress Prob. 47), Vol. II, Birkhäuser, Boston, MA, pp. $249-259$.

[4] Chen, X. (2008). Limit laws for the energy of a charged polymer. Ann. Inst. H. Poincaré Prob. Statist. 44, 638-672.

[5] Chen, X. and Khoshnevisan, D. (2009). From charged polymers to random walk in random scenery. In Optimality (IMS Lecture Notes Monogr. 57), Institute of Mathematical Statistics, Beachwood, OH, pp. 237251.

[6] Chen, X. And Li, W. V. (2004). Large and moderate deviations for intersection local times. Prob. Theory Relat. Fields 128, 213-254.

[7] Chen, X., Li, W. V. And Rosen, J. (2005). Large deviations for local times of stable processes and stable random walks in 1 dimension. Electron. J. Prob. 10, 577-608.

[8] CSÁKI, E., RÉvÉsz, P. AND SHI, Z. (2001). A strong invariance principle for two-dimensional random walk in random scenery. Stoch. Process. Appl. 92, 181-200.

[9] Dembo, A. And Zeitouni, O. (1998). Large Deviations Techniques and Applications, 2nd edn. Springer, New York. 
[10] Fleischmann, K., Mörters, P. and Wachtel, V. (2008). Moderate deviations for a random walk in random scenery. Stoch. Process. Appl. 118, 1768-1802.

[11] Gantert, N., König, W. And Shi, Z. (2007). Annealed deviations of random walk in random scenery. Ann. Inst. H. Poincaré Prob. Statist. 43, 47-76.

[12] Jain, N. C. And Pruitt, W. E. (1984). Asymptotic behavior of the local time of a recurrent random walk. Ann. Prob. 12, 64-85.

[13] Kesten, H. and Spitzer, F. (1979). A limit theorem related to a new class of self-similar processes. Z. Wahrscheinlichkeitsth. 50, 5-25.

[14] Khoshnevisan, D. And Lewis, T. M. (1998). A law of the iterated logarithm for stable processes in random scenery. Stoch. Process. Appl. 74, 89-121.

[15] LEwIS, T. M. (1992). A self normalized law of the iterated logarithm for random walk in random scenery. J. Theoret. Prob. 5, 629-659.

[16] LewIs, T. M. (1993). A law of the iterated logarithm for random walk in random scenery with deterministic normalizers. J. Theoret. Prob. 6, 209-230. 